\title{
Coronary Reperfusion Procedure
}

National Cancer Institute

\section{Source}

National Cancer Institute. Coronary Reperfusion Procedure. NCI Thesaurus. Code C100067.

Procedures intended to re-establish normal coronary flow. Reperfusion therapy includes thrombolysis and primary percutaneous coronary intervention (PCI). Reperfusion therapy also includes per-hospital thrombolytics. (ACC) 